\title{
Numerical Simulation and Experimentation of Additive Manufacturing Processes with Polyurethane Foams
}

\author{
Elodie Paquet ${ }^{1(\otimes)}$, Sébastien Le Loch ${ }^{1}$, Benoit Furet ${ }^{1}$, Alain Bernard ${ }^{2}$, \\ and Sébastien Garnier ${ }^{1}$ \\ ${ }^{1}$ University of Nantes, Laboratoire LS2N (UMR UMR6004), IUT de Nantes, \\ 2 Avenue du Professeur Jean Rouxel, 44470 Carquefou, France \\ elodie.paquet@univ-nantes.fr \\ 2 Ecole Centrale Engineering School of Nantes, Laboratoire LS2N (UMR UMR6004), ECN, \\ 1 Rue de la Noë, 44300 Nantes, France
}

\begin{abstract}
Foam Additive Manufacturing (FAM) is the additive manufacturing process allowing parts to be obtained by depositing layers of polyurethane foam using a high-pressure machine. This inexpensive technology allows large parts to be produced in a reduced time. However, the quality of the parts produced by the FAM technique is greatly affected by the various thermal phenomena present during manufacturing and by the geometrical deviations of the layers due to the expansion of the PU foam. Numerical simulation remains an effective analytical tool for studying these phenomena. The aim of this work is to build a geometric and thermal model predictive of the FAM process by the finite element method, the final objective of which is to provide temperature maps throughout the manufacturing process and also to choose the best 3D printing strategy to have a model with constant cords and the smallest possible form deviation. The proposed model and the various simulation techniques used are detailed in this article. This model is developed under the finite element code Rem3D, and validated by experimental tests carried out on a FAM machinery or a robot, an example of which is detailed in this article.
\end{abstract}

Keywords: Foam Additive Manufacturing $\cdot$ Simulation processes $\cdot 3 \mathrm{D}$ printing

\section{Introduction}

The FAM (Foam Additive Manufacturing) is an additive manufacturing (AM) process a process using an expanding polymer. This technology allows the production of large parts such as nautical tools, by superimposing the layers of material. It represents an interesting alternative to traditional machining processes. Indeed, it is a process which makes it possible to save significant production times in the case of the manufacture of parts with complex geometries and to limit the losses of raw material. The material used is a PU foam shear thinned reacting polymer mixture of adequate isocyanate and polyol group. The foam is deposited on a support with a free expansion where after a few seconds 
the material evolves from a low molecular weight emulsion (through polymerization with the evolution of heat and $\mathrm{CO}_{2}$ gas) to a complex polymer [1]. Compared with other FA processes such as Fused Deposition Modeling process with plastic materials, the FAM is characterized by a high deposition rate (with, in our case, a flow of $43 \mathrm{~g} / \mathrm{s}$ ) because the foam used expands by $30-40$ times its volume in a few seconds. The process also has the capacity to manufacture large parts as well as tools since the quantity of material required is deposited and there is no recycling to be expected. For example, the cost of manufacturing a large tool is less expensive by additive manufacturing than by traditional methods [2]. However, the FAM process also has certain non-negligible drawbacks: during successive deposits, the strong thermal gradients generated by the polyurethane foam which is an insulating material lead to the generation of internal residual stresses, and to deformations in the final part. These residual stresses have an impact both on the mechanical properties (fatigue behavior for example) and on the final geometry of the part and also on the material health of the part (appearance of cracks, burning of material, etc...). These constraints and deformations are directly linked to process parameters such as for example the speed of deposit, the height of deposit, the angle of deposition, the temperature of the components, the flow rate and the time expected between the printing of each layer. The influence of these parameters and the stacking of the cords are evaluated using a thermo mechanical simulation taking into account a Navier Stokes' behavior law for the polyurethane foaming (Rem3D software).

\section{Foam Polyurethane Characterization}

The material of interest here is polyurethane foam. The polyurethane is a polymer bicomponent material obtained by mixing together isocyanate and polyol. It begins to cure in $6 \mathrm{~s}$, expands to nearly 40 times its initial volume to yield a low density of $36 \mathrm{~kg} / \mathrm{m}^{3}$. Its compressive strength is $110 \mathrm{kPa}$, tensile strength is $171.5 \mathrm{kPa}$. The reactions taking place in PU foams are complex, but can be represented by only two global exothermic reactions: the first gelling reaction, where polyol and isocyanate react to form highly cross-linked polyurethane and blowing reaction where water and isocyanate react to form urea and carbon dioxide [3].

In order to model mechanical and thermal reaction, we use modelling software called Rem3D. This software computes the flow of the material by solving the Navier Stokes and the thermal equations.

The material used is very reactive and therefore cannot be characterized in the laboratory. Therefore a characterization of the material has been carried out directly on our robotic cell using a thermocouple and camera. The purpose of the experience is to recover the temperature present during the reaction as well as the material's cooling curve and the expansion heights as well as the weight losses of the material during expansion linked to the evolution of gases present during the mixing reaction.The protocol of the characterization implemented (Fig. 1) recovers the reaction temperature, the climb height, the expansion speed as well as the pressure exerted during the polymer foaming. To carry out the characterization, we programmed a casting of material with a programmed mass of $54 \mathrm{~g}$ over a translucent container with a diameter of $85 \mathrm{~mm}$ fitted with three thermocouples. A camera films the expansion and by image processing, we 
were able to recover the climb height as well as the speed. Another camera films the mass and the pressure difference during casting. To have a correct characterization, we did the handling and processing twice the two experiments: Experiment 1 and Experiment (Fig. 3) in order to average the results and then translate them into digital material file for the REM3D software.

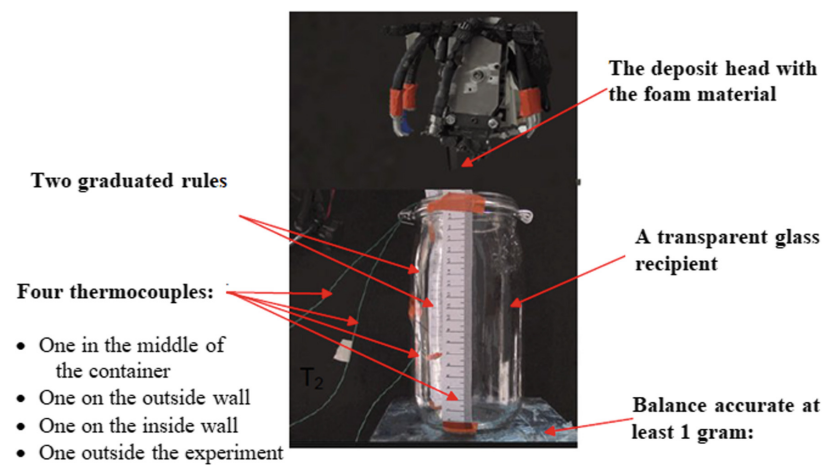

Fig. 1. Characterization protocol for foams with high reactivity in situ.

The first physical parameter measured was the reaction temperature (Fig. 3). Temperature maximum of $178^{\circ} \mathrm{C}$ in the heart is reached in $146 \mathrm{~s}$ and the cooling rate is $2.86{ }^{\circ} \mathrm{C}$ per minute. The total cooling time for a volume of $0.0989 \mathrm{~m}^{3}$ of foam poured is $4096 \mathrm{~s}$. The second physical parameter measured and analyzed by image processing is the expansion rise height as well as the expansion speed measured at $32.3 \mathrm{~mm} / \mathrm{s}$. Once the material has been characterized [4], the data from the experiment (Fluid Flow rate, Height of deposition, speed....) have been translated into a digital material file and implemented in the Rem3D software. Then, the simulation was realized in order to correlate the data from simulations carried out and experiments (Fig. 2 and Fig. 3).

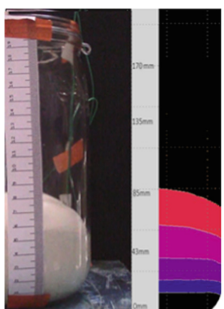

(a)

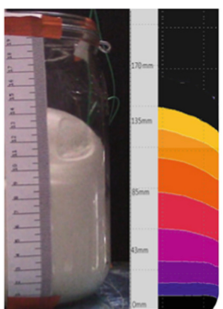

(b)

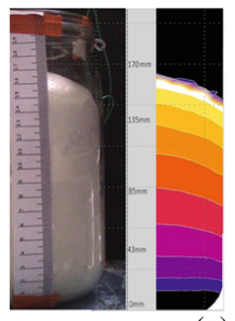

(c)

Fig. 2. Development of the flow front of the foam material in the cylinder with a $85 \mathrm{~mm}$ diameter after $2 \mathrm{~s} \mathrm{(a),} 15 \mathrm{~s}$ (b) and $90 \mathrm{~s}$ (c): results from simulation (right) and practical tests (left)

The compared results are: the volume of foam and the temperature (Fig. 3). The foam volume between simulation and experiment at each time is the same $(a, b, c)$. If we 
compare the reaction exothermal, the maximum temperature in simulation is reached at a time equivalent to the tests. The reaction seems to be faster when start-up.

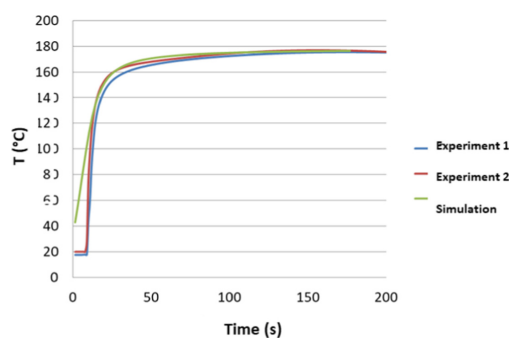

Fig. 3. Time evolution of the temperature in the cylinder in simulation and during experiments

\section{Comparison Between Experiments and Numerical Simulation}

The simulation of the behavior of the material during the layer deposition allows obtaining essential information such as the geometry of the manufactured layer [6], the temperature inside the material, as well as the pressures exerted.

This information is then used to determine the best strategies for the printing process to guarantee the correct geometry of the final part and correct material health. As shown in Fig. 4, it is then possible to compare the simulated image to the image taken from a printed layer section. This comparison makes it possible to highlight any differences in terms of distortion between the experiment and the simulation. Observations show a very good correlation between simulation and experience when depositing several layer.

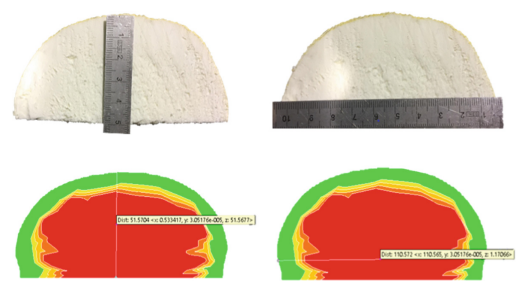

Fig. 4. Example of correlation between simulation and experimentation on a layer printed with a $43 \mathrm{~g} / \mathrm{s}$ rate of flow and a speed deposition of $10.2 \mathrm{~m} / \mathrm{s}$.

\section{Use of Simulation to Apply a Speed Correction on a Trajectory in Order to Minimize the Form Defect}

When manufacturing a part by $3 \mathrm{D}$ printing, if one wants to obtain the correct geometry of the desired bead, it is essential to master certain physical parameters. In order to 
compensate for the geometric drifts of the printed layers, a servo-control in position of the upper layers and in speed was set up in order to achieve and maintain a desired geometry using a direct measurement from a contactless sensor and using the results from the simulation to determine the coefficients $a_{\text {proc }}$ and $b_{\text {proc }}$ to be applied in the relation of the speed of the material deposition to correct speed and smooth out flaws (Fig. 5).

The speed to be applied is given by the equation: $v=\varepsilon * a_{\text {proc }}+b_{\text {proc }}$ (per layer).

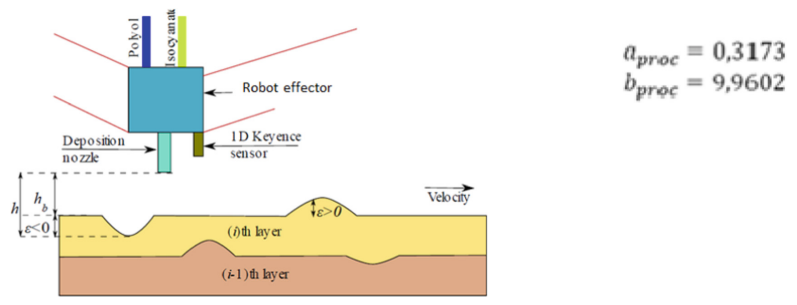

Fig. 5. The principle of the closed loop manufacturing system that has been used

The implementation of this "closed loop manufacturing system" applied to 3D printing [5] aims to better control the deposition of the layers and the final surface condition of the printed part to minimize the potential stages of completion.

Figure 6 shows on the left the surface state of printed layers with position and speed control and on the right the speed profiles associated with the surface states. The enslavement implemented makes it possible to attenuate and compensate for surface defects between the printed layers in order to get as close as possible to the theoretical geometric shape.

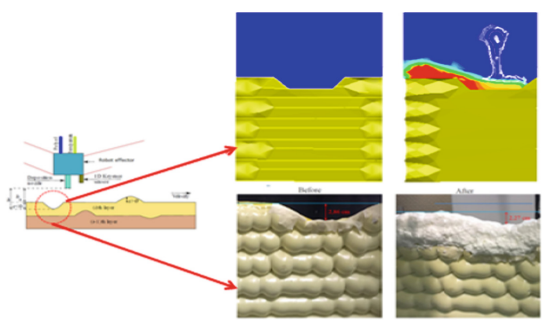

Fig 6. Deposition improvement from $2,86 \mathrm{~cm}$ to $2,27 \mathrm{~cm}$, which corresponds to $20 \%$ in terms of deposition accuracy

\section{Perspectives}

When printing foam of a right angle or a curve with weak radii, the robot slows down on the trajectory and this causes an addition of material in certain area thus creating inhomogeneous layers [7]. These additions of material thus create significant defects 
in shape deviation on the global model. The choice of strategy in terms of trajectories to be carried out at these locations is currently complex and empirical. The simulation could help us to find the optimal parameters of the process which makes it possible precisely to be able to realize these complicated printing zones and thus to optimize this set of defects (non-homogeneous layers, the recovery of layers, the end of layers which is crushed, the layers bursting...). The digital simulation of material deposition from the different operating parameters allows us to make a comparison of the best strategy to choose to ensure the geometric shape to be obtained at the end. Each time, we carry out several simulations, and we come to choose the best by making a comparative analysis of the simulations with regard to quality and productivity criteria.The knowing of thermal comportement permit us to validate a global printing in a complexe workpiece and making sure to have a correct material health of the part after printing.

\section{Conclusion}

In this paper, the potential to use foam simulation for 3D printing of a large scale part was introduced. The use of process simulation to choose the best strategy has been shown across an example of closed loop manufacturing system on a printed part. The process constraints and the development of specific strategy for printing specific area in large parts must be studied in more depth.

Acknowledgment. This work was carried out in the LS2N laboratory at the University of Nantes. The author acknowledges the significant contributions to this paper by the following people: Mme. Sana BAKLOUTI and Mr. Joachim MARAIS at the University of Nantes and is also grateful to TRANSVALOR society for their assistance and technical expertise.

\section{References}

1. Keating, S.J., Leland, J.C., Cai, L., Oxman, N.: Toward site-specific and self-sufficient robotic fabrication on architectural scales. Sci. Robot. 2(5), 15 (2017). https://doi.org/10.1126/scirob otics.aam8986

2. Poullain, P., Paquet, E., Garnier, S., Furet, B.: On site deployment of 3D printing for the building construction-The case of Yhnova TM. In: MATEC Web of Conferences, vol. 163, no. 5, p. 01001, January 2018. https://doi.org/10.1051/matecconf/201816301001

3. Barnett, E., Gosselin, C.: Large-scale 3D printing with a cable-suspended robot. Addit. Manuf. 7, 27-44 (2015). https://doi.org/10.1007/s41693-017-0008-04

4. Rossen, W.R.: Numerical challenges in foam simulation: a review. In: Society of Petroleum Engineers (2013). https://doi.org/10.2118/166232-MS. (Delft University of Technology)

5. Barnett, E., Angeles, J., Pasini, D., Sijpkes, P.: Surface mapping feedback for robot-assisted rapid prototyping. In: 2011 IEEE International Conference on Robotics and Automation (ICRA), pp. 3739-3744. https://doi.org/10.1109/ICRA.2011.5979689

6. Talagani, F., DorMohammadi, S., Dutton, R., Godines, C., Baid, H., Abdi, V., Brett G. C., Simunovic, S.D., Chad, E., Love, L.J., Post, B.K., Blue, C.A.: Numerical simulation of big area additive manufacturing (3D printing) of a full size car. United States: N (2015) 
7. Baklouti, S., Courteille, E., Lemoine, P., Caro, S.: Vibration reduction of cable-driven parallel robots through elasto-dynamic modelbased control. Mech. Mach. Theory 139, 329-345 (2019)

8. Izard, J.-B., Dubor, A., Hervé, P.-E., Cabay, E., Culla, D., Rodriguez, M., Barrado, M.: On the improvements of a cable-driven parallel robot for achieving additive manufacturing for construction. In: Cable-Driven Parallel Robots, pp. 353-363. Springer (2018)

Open Access This chapter is licensed under the terms of the Creative Commons Attribution 4.0 International License (http://creativecommons.org/licenses/by/4.0/), which permits use, sharing, adaptation, distribution and reproduction in any medium or format, as long as you give appropriate credit to the original author(s) and the source, provide a link to the Creative Commons license and indicate if changes were made.

The images or other third party material in this chapter are included in the chapter's Creative Commons license, unless indicated otherwise in a credit line to the material. If material is not included in the chapter's Creative Commons license and your intended use is not permitted by statutory regulation or exceeds the permitted use, you will need to obtain permission directly from the copyright holder.

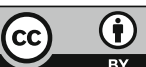

